\title{
Téoros
}

Revue de recherche en tourisme

\section{La villégiature dans Charlevoix : une tradition séculaire, un patrimoine encore vivant}

\section{Philippe Dubé}

Volume 14, numéro 2, été 1995

Le tourisme : toute une histoire!

URI : https://id.erudit.org/iderudit/1075092ar

DOI : https://doi.org/10.7202/1075092ar

Aller au sommaire du numéro

Éditeur(s)

Université du Québec à Montréal

ISSN

0712-8657 (imprimé)

1923-2705 (numérique)

Découvrir la revue

Citer cet article

Dubé, P. (1995). La villégiature dans Charlevoix : une tradition séculaire, un patrimoine encore vivant. Téoros, 14(2), 4-7. https://doi.org/10.7202/1075092ar d'utilisation que vous pouvez consulter en ligne. 


\section{La villégiature dans Charlevoix : une tradition séculaire, un patrimoine encore vivant \\ Philippe Dubé}

$\square$

Le besoin d'un contact avec la naturea été de tout tempschanté par des générations de citadins, à commencer par Pline Le Jeune (Lettres) de sa Rome antique, en passant par Stendahl (Mémoires d'un touriste) jusqu'a Edgar Morin (Vivent les vacances) qui ont tous salué les bienfaits de la cmpagne en lesopposant trop souvent aux méfaits de la ville. L'on remarque dans ces écrits une antinomie abusivement exploitée où se dégage une certaine idée de la nature, en somme une certaine idée de la villégiature. Retrouver la campagne à la ville est un phênomène relativement récent, commesi ces soeurs ennemies - ville et campagne - pouvaient être réunies par un banal compromis. Ceci est une autre histoire que nous n'aborderons pas ici puisque nous nous proposons de définir d'abord la villégiature en termes historiques à partir de critères spécifiquement urbains. Autrement dit, nous tenterons à partar du cas de Murray Bay dans Charlevoix - de bien cerner ce mouvement des villes vers la campagne et de voir en quoi son implantation s'élabore sur la base de courantsémanant d'uneprofonde ur banité. Sur cette lancée, nous suggérons donc comme hypothèse de base que le besoin de campagne vienne de la villeet quece besoin de nature origine d'un besoin de culture, urbaine il va sans dire. Certe dialectique repose essentiellement sur un rapporttendu entre la nature et la culture, et c'est ce que nous allons sommairement examiner ici.

Pour le touriste, la villégiature représente la fin de l'errance et l'architecture de villégiature devient alors l'expression hyperbolique de son établissement au pays des vacances. Au fil des ans, ce symbole dans l'espace prend différentes formes que nous avons voulu regrouper en quatre (4) types, correspondantchacun à quattre temps consécutifs étalés sur une histoire longue deplus d'un siècle et demi. Cette séquence temporelle s'est imposée à nous alors que

Monsieur Philippe Dube est professeur agregé en muséologe a l'Université Laval, Les phologra. phies qui accompagnent le texte sont tirées de son live : Doux cents ans de villeglature dans Chatevolx, Quebec, P.U.L. 1986

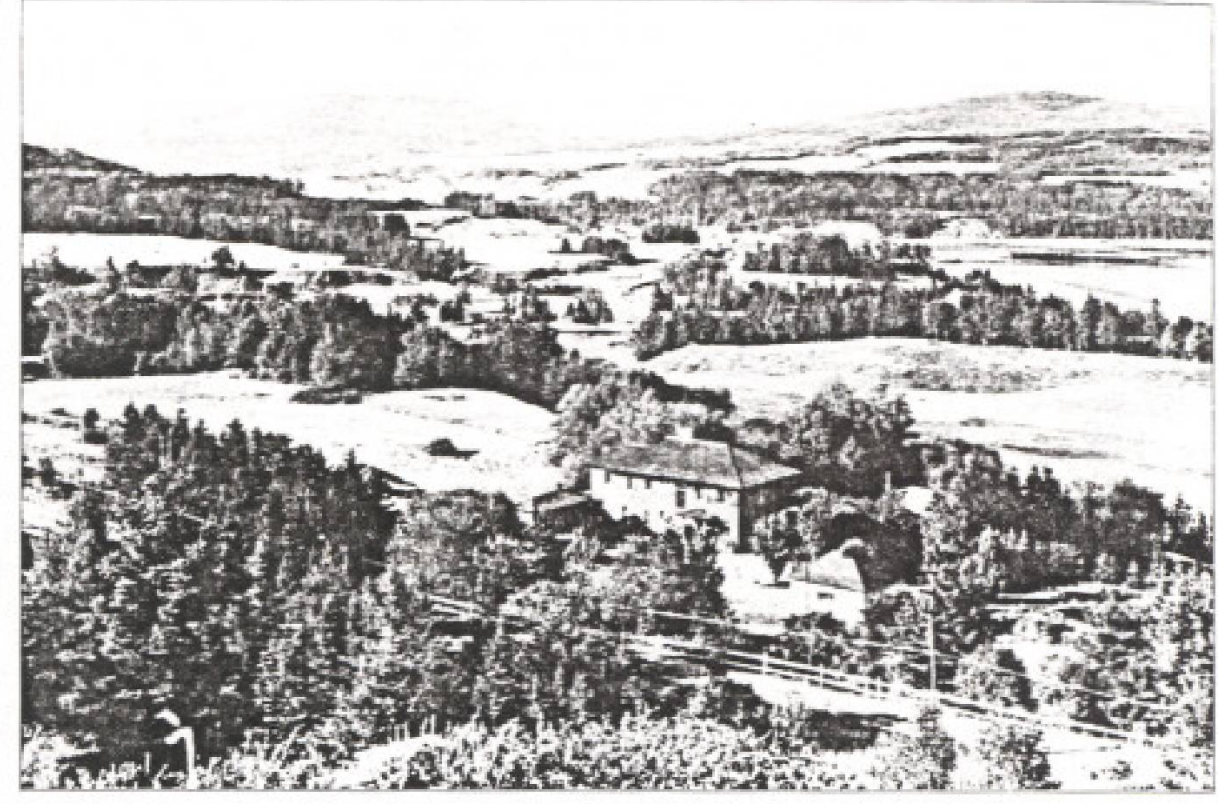

La force du panorama. Nulle théorie ne peut prétendre qu'elle saí, suivant des principes savants, mettre en relacion l' architecture au paysage. Seule une volonté très nette d'empaysement- fait qu"une villa ou un jardin se marie à ce heau pays dans une union durable devant l'eternité. Photo: Miss Louise Crane, collection privée, photographie de =Associated Screen Newsw Ltd, Montréal, CSL175-1, CSL175-2

nous avons tenté d'essayer d'expliquer la villégiature à partir de la ville, et non du point de vue de la campagne. De fait, à chaque phénomène qui vient périodiquement marquer la cité, s'élabore une manière différente de vivre en vacances et, du coup, une façon nouvelle d'installer sa demeure et d'ainsi villégiaturer à chaque fois d'une manière différente. Le séjour saisonnier commande tout d'abord une relation privilégiée avec l'air, puis avec l'eau où la santé du corps domine entre toutes préoccupations. Dans une première phase, la quête de bienfaits thérapeutiques par la villégiature correspond tout d'abord à un besoin d'ordre médicinal où l'équilibre, si l'on veut écologique de l'Homme, est en cause et il s'agit d'y pallier avec urgence. Puis à un autre niveau de besoins s'insère l'idée de panorama qui va dé́placer la priorité du vacancier vers d'autres horizons, celui par exemple d'une conception de plus en plus intégrée du paysagisme, forçant ainsi l'apparition d'une notion aujourd hui acquise mais non moins nouvelle pour l'époque - celle de la vue panoramique. De cette vision moderne de la nature, au sens où il $y$ a rupture radicale entre le corps et l'esprit, va naître peu de temps après une certaine image du pays où le paysage servira à construire le pays. Du bain de mer on passe au bain de rêve où l'imaginairen'a de limite que la valeur de sa pertinence. C'est comme ça que l'écologique devient idéologique pour mieux répondre à l'écho de la ville qui s'étend et se faitencoreentendre dans noscampagnes.

Fuir la ville pendant qu'il en est encore temps

Comme le rappelle si justement Lafontaine dans une de ses fables connues ${ }^{(1)}$ :
Un mal qui répand la terrewr,
Mal que le ciel en sal fureur, Inventa powr punit
les crimes de la terre,
La peste puisqu'il faut Pappeler par son nom,
Capable d'enricbir en un jour IAcheron ${ }^{(2)}$. 
en effet la peste - vocable contenant le paroxysme des maladies contagieuses et épidémiques - est capable de dévaster des villes entières, au moment où l'hygiène publique reste encore en équilibre précaire. Plus près de nous, dans un contexte de colonie en plein essor où l'immigration déborde d'activités intenses dans le port de Québec, des épidémies de choléra viennent périodiquement ravager une population inquiète, autant les immigrants nouvellement débarqués que les résidents de la ville. Le fléau frappe successivement Québec, à partir de 1842 et ce, de manière dramatique. Cet épisode pour une ville relativement jeune est reconnu comme particulièrement sombre dans son histoire. D'autant plus qu'une certaine aisance gagnait peu à peu la bourgeoisie montante qui s'établissait progressivernent dans un pays en train de se faire. Ceci dit, le statut devilleportuairequi amenaituneprospérité certaine à la cité juvénile, faisait avec elle déferler une série de malheurs qui vont marquer pour plusieurs décennies l'imaginaire des gens de Québec. Ses rues insalubres, foudroyées, dévastées par le typhus vont rappeler cruellement à la population de l'époque qu'une vie saine en ville est réellement menacée, pour ne pas dire impossible tant que les conditions d'hygiène ne seront pas mieux respectées. Quand une population immigrante débarque à pleins qua is durant les rares mois navigables de l'été, la ville se trouve littéralement assiégée par une horde d'indigents qui cherchent désespérément refuge à leur misère sans nom. Ce climat de fin du monde frappe la ville entière et tous ceux qui en ont la capacité pensent la fuir obsessivement.

Il fautd'ailleurs se replacer dans un contexte de réelle panique pour bien comprendre ce beroin dair pur que pouvaient ressentir intensémentlescitadins del'époque. Cette première vague de vacanciers était surtout composée de malades, de convalescents, de gens physiquement faibles qui devaient à tout prix récupérer du *mal de la ville» que représentait l'invasion cyclique des immigrants durant la belle saison. Avant la création d'une île de la quarantaine Grosse-Isle en l'occurrence, au large de Monunagny - aménagée pour venir en aide à la population que la contamination menaçait, le citadin qui pouvait se le permettre fuyait la ville pour la campagne afin de se mettre à l'abri d'un mal dévastateur. Cette solution de fuite ne donnait d'autre choix que de gagner une région qui n'était pas encore atteinte par l'insalubrité et qui pouvait encore offrir une nature wierge, immaculée et sans taches». Voilà l'attraction première qui relève davantage de l'instinct de survie parce que l'espèce, cette fois, est réellement menacée. Le premier mouvement de vacanciers sera donc motivé par le goüt de loger chez l'habitant qui veut bien prêter sa demeure en retour de quelques sous. L'habitade rustique devient alors le whome* parfait pour la saison d'été, au beau milieu d'un univers paysan et naturel où l'on espère refaire ses forces. L'aspect campagnard devient tout à coup la valeur recherchée, garante d'une vie saine et paisible, à l'abri des désagréments urbains. Quand le président américain, William Howard Taft, qualifie l'aunosphère de Murray Bay de *champagne of airm, il venait de créer un nouveau cru que ses prédécesseurs villégia teurs avaient découvert bien avant lui. Prendre l'air de la campagne devenait alors une image chargée de sens.

\section{Les eaux miraculeuses}

Au terme de cette première période trouble, de 1840 à 1870 environ, l'on arrive enfin à vaincre les épidémies ravageuses et à «nettoyer les villes de ce mal venu d'ailleurs. D'autres préoccupations vont maintenant tenailler certaines classes urbaines en quête d'un repos bien mérité. Le romantisme, agissant encore dans les décennies tardives du XIX ${ }^{c}$ siècle, de 1870 à 1900 environ, participe à une certaine mystification de la nature et n'arrête de chanter, de glorifier même, ses bienfaits multiples. Ũne vogue extrêmement enthousiaste va littéralement envahir le goût du jour. Cette vogue peut aussi bien être nomméevague puisqu'il s'agit du thermalisme qui s'impose de plus en plus comme le remède à tous les maux. En effet, le bain d'eau salée obtient un tel succès au palmarès des thérapies, que Murray Bay se transforme très rapidement en une station balnéaire de renom qui va bientôt caractériser l'endroit d'une spécificité déterminante, pour ne pas dire indélébile. Cette idéalisation de learu, poussée à l'extrême, promet une authentique résurrection et le bord de mer devient du coup un *musto incontournable pour qui veut soigner ses rhumatismes lancinants, panser ses plaies brûlantes, guérir un mauvais tour de reins ou encore contrer une calvitie galopante. L'eau, source de vie, gagne ainsi ses lettres de noblesse et révèle ses pouvoirs à tous les pèlerins qui croient en elle. Il y a donc un léger déplacement dans l'ordre des bienfaits. L'eau acquiert alors un statut privilégié qui renforce davantage le joug de la nature sur la villégiature, créce essentiellement pour et par les citadins. La petite colonje de La Malbaie va plutôt se développer à partir de la rive et c'est sous un rapport différent que l'acte de villégiature va maintenant se poser. Le front de mer (*ocean frono ) dès lors présidera aux assises des vacances.

Avec une reconfiguration de l'espace champêtre, vont obéir à cette nouvelle prémisse les constructions en bordure du fleuve. L'attrait pour l'élément aquatique fera se rapprocher les vacanciers qui veulent se confronter à l'eau. Cette volonté de contact avecla partie fluviale dela région va surtout être manifeste chez les Américains qui tuansposeront simplement la réalité balnéaire de la Nouvelle-Angleterre (les plages du Maine et du Massachussetts sont déầ très prisées) à un site maritime qui se prête, pour un temps, à ce jeu de mascarades. Certe manière américaine s'inspire directement des traditions développées en bordure de la Manche, soit du côté anglais avec Brighton en tête de liste ou encore sur la côte normande, avec Trouville et Deauville comme chefs de file français. Cette villégiature balnéaire commande un pavillon bien intégré au contexte océanographique et les formes qu'il va épouser fera de lui un solide loup de mer. Ses traits auront les lignes souvent arrondies du whingle style» - tel que nommé par Vincent Scully - qui se fonderont habituellement à l'esprit du lieu. Le profil parfois «archéologique», comme pouvait le qualifier à l'époque l'observateur averti, correspond parfaitement à ce goùt pour une certaine renaissance ( tisée *americana» et qui dominait les goûts culturels denos voisins du sudà ce momentlà. Les formes architecturales traditionnelles américaines étaient utilisées à cette fin comme pour emprunter l'audace et la foi enthousiaste des premiers immigrants qui sont venus s'implanter en terre d'Amérique. Cette référence à la genèse de la colonisation s'inscrit parfáitement sur les bords d'un plan d'eau de mer à qui l'on prête un pouvoir médicinal sans avoir pour autant uneidée très précise deses bienfaits. Cette aura va marquer non seulement une époque mais aussi une rive par un băti qui cherche effrontément un face-à-face avec la mer. 


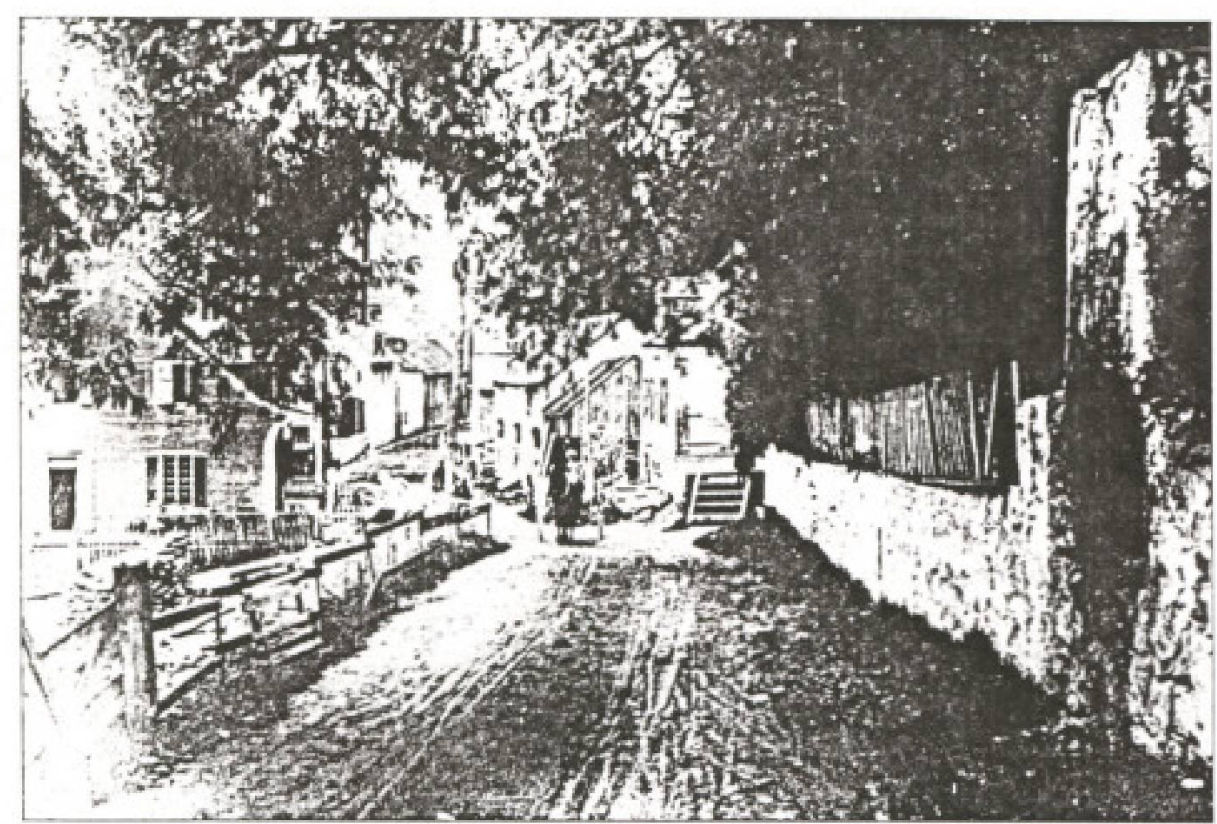

Murray Village. Certaines maisons ancestrales etant de beaux exemples d'architecture domestique, scrviront de modetes a plusieurs villas. Cette scène da village de La Malbaie, croquete par Notman, photographe répute de Montréal, traduit avec jutesse l'émotion pittoresque tant recherchée au tournant du siecle.

Photo : Collection Archives photographiques Norman, Mustée McCord, Montríal, $32 k 6$, sous le tite - Murray Bay Villagem, vers 1900.

\section{Le spectacle de la nature}

De ce rapport étroit avec le système écologique - air et eau - va naitre une toute nouvelle tendance dans la manière de s'approprier un territoire qui n'a pas encore été souillé par l'activité urbaine. Depuis que thygiène publique est sous le contröle des autorités civiles, vers les années 1895-1898, l'insalubrité des villes est finalement maîtrisée par des mesures strictes qui font que la tie en grand nombre sur une surface relativement petite est maintenant rendue possible, pour ne pas dire viable. Finis les dangers de contamination à cause d'une concentration trop forte de population, voilà que la ville en tant qu'espace de vie collective ne menace plus la santéphysique des gens qui thabitent. Toutefois va poindre un autre besoin qui trouve encore une fois son origine dans les dédales de macadam. D'un certain romannisme où la nature est ouvertement glorifiéeva découler tout bonnement un goût pour les paysages que l'on nommerait, pour la convenance, wpaysagismes, c'est-à-dire l'école qui encourage la composition artistique prenant pour theme principal la nature. Ce toisième déplacement, de 1900 à 1935 environ, succede aux autres par une operatoon assez singulière qui fait passer la villégiature, en tant que phénomène urbain, d'un ordre primaire ('équilibre physique) à un ordre socondaire ('équilibre mental).
En effet, cette recherche obsunée de la *belle vuew va devenir à ce point dominante que l'implantation du bätiment sur le site sera fonction uniquement du point de vue qu'il offre aux résidents devenus aussi observateurs et spectateurs. L'architecture sera alors mobilisée par l'intégration du belvédère dans la volumétrie qu'elle suggère. La forme qui tente de répondre adéquatement à la fonction quilui est confiée épouse non seulement un emplacement nouveau mais aussi une configuration inhabituelle qui se doit d'être adéquate.

Ceparti-pris pour lepanorana exige donc un langage formel plus êlaboré qui doit manier avec aisance la rencontre desverticales et des horizontales. Chaque geste architectonique esi question de mesure, de rythme, et de stemposs pourrait-on dire. Les espaces sont élahorés en rapport non plus direct avec la narure, comme l'air et l'eau, mais indirectement cette fois avec l'immensité du paysage offert à la vue. L'idée de se haut percher est absolument obligée dans une démarche de mieux voir, d'arriver doncà trouver lesite qui rếpondra le mieux à la projection visuclle que l'on veut offrir. Cette orientation dirigée vers une nouvelle manière d'occuper l'espace force le concepteur à des prouesses techniques qui permeturont l'évolution d'une gestuelle volumétrique novatrice. Cet art de bâtir poussera un peu plus loin les limites du construit et offrira à l'esprit curicux un laboratoire intéressant pour l'expérimentation créatrice. Une des règles fondamentales vient de s'écrire pour toujours en ce qui regarde le villégiateur, à savoir la distance d'ores et déjà nécessaire pour apprécier ce qui s'étale sous ses yeux. Le recul devient alors le passage obligé à une mise en espace de la nature, stratégie scénographique incontournable pour offrir en plongée les plus beaux paysages. Point de vue est le mot-clé de cette grammaire quil apportera un déferlement de figures aux con tours parfois capricieux. $\mathrm{La}$ retraite n'est plus une défaite dans cecorpsà-corps avec la nature mais prendra plutôt l'allure d'un tête-à-tête où l'esprit se grise de plaisirs transitant par l'oeil insatiable du regardeur qui cherche obsessivement l"effet visuel.

\section{À la recherche d'un pays imaginaire}

Du terme paysage qui se définit comme «une étendue de pays $\%$, il est tentant d'en revenir à sa racine, non pas pour le simple jeu de mots, mais parce qu'il est un dérivé réel du terme spays $\%$. Si sa composition sémantique exprime cette réalité profonde, il faut comprendre que le paysage englobe virtuellement le pays. Au-delà de la simple représentation d'un site généralement champetre que nous offre le paysage, il peut contenir sinon le pays lui-mëme, à tout le moins l'idée que l'on s'en fait. En effet, le tableau d'une nature indigène peut en soi renfermer lemicrooosme d'uneréalité beaucoup plus vaste et ce, de façon abstraite. Dans la figuration d'une sorte de beauté picturale, l'imaginaire peut se surprendre à projeter sur l'écran flou de la nature une vision du pays, surtout si ce dernier est en train de se définir politiquement. Decetordre fantasmatique, où il s'agit d'une représentation par l'imagination, va découler tout un lytisme qu'entretient la recherche désespérée de l'ici en tant qu'entité nationale. Cé désir du pays qui se consume dans l'image que le paysage nous renvoie annonce le dernier ìge de ce quatuor, à partir de 1935 jusqu'a 1965 environ, où l'insertion harmonieuse à l'espace rend plus réelle cette appartenance encore fragile qu'offre le Canada. II faut bien comprendre que ce mouvement se propage surtout parmi la classe des intellectuels canadiens-anglais qui vit tragiquement la conscience d'une absence d'identité culturelle de plus en plus difficile à 
supporter. Le paysage canadien - et celui de Charlevoix particulièrement-devient alors un pồle majeur de définition figurative du Canada ou le territoire se travestit volonters, pour les besoins de la cause, en un emblème national signifiant que l'on doit, somme toute, porter haut. Il s'agit d'un hymne à la beauté que l'on entonne avec ferveur, comme pour entretenir une flamme vibrante envers la patrie.

Cet élan nationalitaire qui relève du processus maintenant connu sous le nom de *nation buildings se traduit tout d'abord par une mode mimétique où la fidélité à la tradition crée le genre *antiquairem que l'on bapusera «canadianass. L'architecture de villégiature qui endosse ce credo va tout d'abord concevoir son image du pays dans le bâti comme une copie conforme de l'ancien. L'intégrité formelle ne réussit pas toujours à contenir les besoins grandissants d'une vie moderne, mais on revêt de matériaux rustiques les formes traditionnelles afin de préserver intacte une facture *canadiennes. Ce premier réflexe d'imitation sera suivi d'une démarche beaucoup plus actuelle oủ l'imagination s'intègre plus librementaux volumes d'antan. Il s'agit d'un retour au passé qui se projerte uldimement dansl'avenir et cepassageau passéantérieur permet une avancée, un peu timide certes, mais une percée tout de mêmevers le futur. Les oeuvres qui se réclament de cette volonté s'implantent indifféremment sur le bord du fleuve ou sur un plateau, ou encore s'enfoncent dans un boisé touffu. Chacune est porteuse d'une recherche compulsive du pays idéel oú le dialogue avec l'environnement, en tant qu'interlocuteur valable, est par dessus tout privilégié. Voilà qui est résolument utopique, au sens premier du terme, car l'on croit fermement, et quelque peu naivement, prendre racines en ce pays en cherchant l'accord parfait, l'harmonie totale avec un milieu physique qui a longtemps rimé avec misère. Cette mise en rapport avec le pays, se servant du courant nationaliste canadien pour mieux se brancher sur une réalité encore difficileà cerner, relève d'un monde oniriqque qui vient à la rescousse d'une souffranoe plus sourde mais qui ne fait pas moins mal. Ce mal à l'âme conadienne meut encore nombre de nos concitoyens. L'architecture est ici archi/ texturé d'une réalité historique et politique que nous devrons tôt ou tard dépasser.

\section{À bout de souffle}

Cette valse à quatue temps nous instruit en fait sur un ordre qui, bien que séquentid, est cumulatif au sens où les besoins comme les bienfaits de chaque äge sont récurrents et restent aujourd'hui présents au rang des préoccupations et des motivations des estivants. La villégiature rustique, comme la balnéaire ou encore la panoramique sont toujours actives en tant que manières d'être en vacances. $\mathrm{Par}$ ailleurs, l'aspect identitaire - au sens de quête d'un trait collectif et national - est beaucoup moins perceptible a ujourd'hui, compte tenu vraisemblablement du contexte politique désta bilisant et pietinant de l'heure. Finalement, nous avons affaire à un cycle qui tente, ces dernières années, de se refaire sans cesse, selon le rythme d'une sfigure de Frasers qui, d'après l'historien Jacques Attali (o), est l'emblème du progrès continu représentế par la spirale. Les quatre saisons d'une villégiature accumule, en définitive, les parfums et les charmes de chacune dans un espace-temps qui s'étale éperdument.

Pline qui appréciait particulièrement passer ses vacances à Lac de Come, dans le nord de l'Italie actuelle, avait su tirer le meilleur parti des qualités de là-bas en se faisant construire deux villas, l'une au bord du lac, l'autre accrochée en flanc de montagne. Il partageait ainsi son temps de repos entre deux résidences qui, chacune à leur manière, apportaient sa part de bonheur. Comme pour mieux qualifier ce qu'il retirait de ces lieux distincts, il avait nommé celle qui longeair la rive de l'eau aComédien, alors que l'autre portait le nom sublime de aTragédiew. Dans la *Comédies où il aimait s'abandonner, tout était léger, calme et translucide, fluide en fait comme l'eau qui s'étalait devant elle. Par ailleurs, quand il se réfugiait dans la *Tragédies, tout devenait quelque peu dramatique. $\mathrm{La}$ seule vue du paysage introduisait une solennité qui exacerbait la dimension théătrale du espectacolom. C'est dire combien il y a de manières différentes d'étre en vacances et qu'ici nous avons tenté d'en définir quatre, à partir des bescoins sentis et exprimés par la ville. Reste à savoir si l'on peut indéfiniment multiplier les genres et en arriver à faire une typologie exhaustive des sortes de villégiature. A cet égard, que peut-on attendre du XXI siècle comme nouveauté en certe mațère? Est-ce que la dichotomie entre la ville et la campagne sera toujours aussi nettement tranchée? Car on sait maintenant que la ville a sans gêne inféodé la campagne sur le compte de la logique de ses propres besoins. Peut-on s'attendre à ce que la campagne vienne un jour transformer la ville comme cette dernière l'a fait envers la campagne à titre de métropole fantasque qu'elle était? Nous nous risquons à penser que les frontères entre les deux mondes - ville et campagne - seront de moins en moins étanches et que pour participer à un développement optimal, de part et d'autre, il faudra tirer le meilleur de chacun sans toutefois les dénaturer tous les deux.

Charlevoix a, pendant près de deux siècles, joué le rôle gratifiant d'offrir un monde meilleur à ceux qui voulaient se revivifier le corps et l'esprit. Demain qu'est-ce qui lui adviendra, maintenant que ses compétteurs directs sont les Iles Séchelle, quelques paradis perdus de la Polynésie ou encore les prés verts et vallonneux d'Irlande? $\mathrm{A}$ lons-nousà nouveau créer et construire des résidences secondaires alors que la plupart d'entre elles servent aujourd'hui d'auberges? Voilà autant de questions qui méritent des réponses et nous fon t conclure que la seule façon de pressentir les demains de la villégiature est d'observer très attentivement les citadins dans leur quette de repos et de tenter de voir si, dans l'avenir, ils trouveront mieux que d'aller se faire rồtir sous un soleil doré comme des lézards épuisés qui échouent sur une plage oiseuse. Sans contredit, l'archéologie de la villégiature dans nos belles campagnes nous mene directement et profondément au coeur de la ville. Sous les pavés, il y a effectivement la plage, comme l'exprimait si bien le cêlèbre graffiti de mai 1968. A nous d'en révéler less sédiments - comme on l'a vuencore profonds. $f$

\section{NOTES}

(11) Jean de Lafontaine, Les anima Lx malades de la peste, Uvre VII, fable 1.

(2) Acheran: fleuve des enters que les ämes des morts, condutes par le oocher Charon, traversaient sans retour.

(3) ATTALI. Jacques, La figure de Fraser, Paris, Fayard, 1984. 